\title{
Evaluating the profound effect of gut microbiome on host appetite in pigs
}

\author{
Hui Yang ${ }^{1,2+}$, Ming Yang ${ }^{3 \dagger}$, Shaoming Fang ${ }^{1}$, Xiaochang Huang ${ }^{1}$, Maozhang He ${ }^{1}$, Shanlin Ke', Jun Gao', \\ Jinyuan $\mathrm{Wu}^{1}$, Yunyan Zhou', Hao Fu', Congying Chen ${ }^{1 *}$ (D) and Lusheng Huang'
}

\begin{abstract}
Background: There are growing evidences showing that gut microbiota should play an important role in host appetite and feeding behavior. However, what kind of microbe(s) and how they affect porcine appetite remain unknown.

Results: In this study, 280 commercial Duroc pigs were raised in a testing station with the circadian feeding behavior records for a continuous period of $30-100 \mathrm{~kg}$. We first analyzed the influences of host gender and genetics in porcine average daily feed intake (ADFI), but no significant effect was observed. We found that the Prevotella-predominant enterotype had a higher ADFI than the Treponema enterotype-like group.

Furthermore, 12 out of the 18 OTUs positively associated with the ADFI were annotated to Prevotella, and Prevotella was the hub bacteria in the co-abundance network. These results suggested that Prevotella might be a keystone bacterial taxon for increasing host feed intake. However, some bacteria producing short-chain fatty acids (SCFAs) and lactic acid (e.g. Ruminococcaceae and Lactobacillus) showed negative associations with the ADFI. Predicted function capacity analysis showed that the genes for amino acid biosynthesis had significantly different enrichment between pigs with high and low ADFI.
\end{abstract}

Conclusions: The present study provided important information on the profound effect of gut microbiota on porcine appetite and feeding behavior. This will profit us to regulate porcine appetite through modulating the gut microbiome in the pig industry.

Keywords: Pig, Feed intake, Gut microbiota, Enterotype, 16S rRNA, Function prediction

\section{Background}

Feelings of hunger and satiety are the principal involuntary motivations for animal feeding behavior [1]. The feeding behavior mainly includes two phases: the appetitive phase (e.g. foraging) and the consummatory phase (e.g. chewing and swallowing) [2]. The appetitive phase brings animals to contact with food, whereas the consummatory phase is the final reflexive response. Both of these phases can increase the hunger, the desire of appetite and food intake. Therefore, it is important to elucidate when to seek food, what to eat and how to eat. There are two models for appetite control: one is involved in host energy

\footnotetext{
* Correspondence: chcy75@hotmail.com

${ }^{+}$Hui Yang and Ming Yang contributed equally to this work.

${ }^{1}$ State Key Laboratory of Pig Genetic Improvement and Production

Technology, Jiangxi Agricultural University, Nanchang 330045, China

Full list of author information is available at the end of the article
}

homeostasis and hedonics; the other model is based on bacteria-host communications [1].

The homeostatic model reflects the balance of energy intake and expenditure in the host. The model explains that appetite is triggered by energy shortage. The center for homeostatic control of energy balance is located in the hypothalamus, which plays a key role in integrating energy information through neural and humoral pathways [3]. The neural pathway senses peripheral energy status via vagal afferents, which relay the nutritional information to the solitary tract nucleus in the brainstem and subsequently transmits to the hypothalamus. The humoral pathway directly circulates nutrients and hormones (such as glucagonlike peptide 1 (GLP-1), peptide YY (PYY), insulin and leptin) to the hypothalamic arcuate nucleus (ARC). Within the hypothalamus, pro-opiomelanocortin (POMC) neurons regulate satiety, whereas neuropeptide tyrosine 
(NPY) and agouti-related peptide (AgRP) neurons are involved in hunger signaling. The POMC, NPY and AgRP neurons are often regarded as the 'first order' in the pathways of hunger and satiety [4]. When animals increase appetite and food intake, the feeding-related feeling of pleasure might underlie the hedonic reason for eating. An abnormal hedonic driving for eating can override homeostatic signals and cause eating disorders, such as bulimia nervosa [5] and obesity [6].

Accumulating evidences indicate that the gut microbiota plays an important role in the bidirectional communication between the brain and the gut $[1,7]$. In particular, the microbiome in the gastrointestinal tract has an important influence in host energy metabolism. For instance, dysbioses of the gut microbiota has contributed to kwashiorkor [8], obesity [9] and anorexia nervosa [10]. More and more studies have indirectly demonstrated the role of the gut microbiota in host eating behavior. Zhang et al. [11] found that the gut microbiota could be shifted by changing the amount of food intake. Breton et al. [12] showed that gut bacterial proteins could activate host satiety pathways and have an effect on host control of food intake dependent on the bacterial growth cycles. The optimal regulation of host appetite might be ensured by both bacteria derived chemical signals and the energy status of the gut microbiota. The molecules produced by gut bacteria should be detected by the chemical sensory elements of the gut epithelium located on enteroendocrine cells, and then activate the pathways of appetite control [13]. However, these hypotheses about how the gut microbiota influences the host appetite remain uncovered. There are few studies about which microbes and how the gut bacteria regulate host feeding behavior. Furthermore, the causality between the change of the gut bacteria and host feeding behavior remains to be established.

The aim of the present research is to explore the potential impact of gut bacteria on porcine feeding behavior using $16 \mathrm{~S}$ rRNA gene sequencing, and to identify the possible bacterial taxa influencing porcine appetite. As pigs have been used as a biomedical model for human diseases, the identification of bacterial molecules involved in feeding behavior could also provide useful reference for the prevention and treatment of human eating disorders.

\section{Results}

Phenotypic characteristics of porcine feeding behavior

The phenotypic distributions of feeding behavior traits including average daily feed intake (ADFI), average daily eating time (ADET) and average daily eating visits (ADEV) in the experimental cohort are shown in Additional file 1: Figure S1. The phenotypic values basically fitted the normal distribution. As expected, the
ADFI had a significantly positive correlation with backfat $(r=0.41)$, average daily gain $(r=0.58)$ and residual feed intake $(r=0.60)$, and the ADET was positively correlated with the ADEV. However, there was no significant correlation between ADFI and ADET, and between ADFI and ADEV (Fig. 1). We then analyzed the effects of gender, host genetics and pen on the phenotypical values. Host gender and genetics (full-sibs vs. unrelated individuals) showed no significant influences in the phenotypical values $(P>0.05$, Additional file 2: Figure S2). However, pen had a significant effect on phenotypes. Pigs in different pens were detected to have significant differences of ADFI values by pairwise comparison (Additional file 3: Table S1). Besides, the pigs in the same pen exhibited more similarity of appetite (Additional file 2: Figure S2). Interestingly, our previous study also found that the diversity of gut microbiota in the pigs housed in the same pen was more similar than that in the pigs in different pens [14]. We assumed that the more similarity of gut microbial composition resulted in the similarity of appetite for pigs in the same pen.

\section{Enterotypes and its association with porcine feeding behavior}

We obtained 11,709,234 sequence reads in 280 fecal samples. After clustering based on the $97 \%$ similarity, an average of 759 OTUs for each sample was identified.

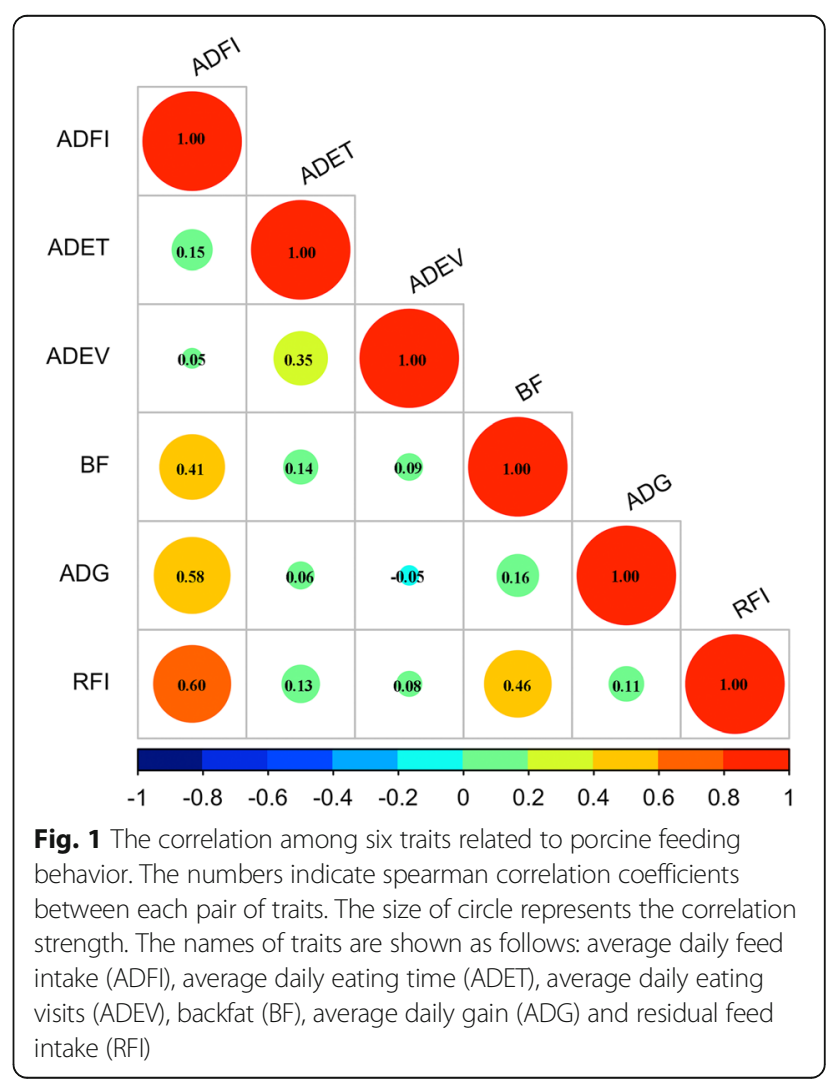


Consistent with the previous reports $[15,16]$, Bacteroidetes and Firmicutes were the most abundant phyla in the fecal microbiota of pigs. But significant difference existed in the relative abundances of bacterial taxa among 280 samples, suggesting the animal-to-animal variation of the phylogenetic composition. We classified the enterotypes for all tested samples according to their phylogenetic composition via the method described by Arumugam et al. [17]. PCA analysis was used to observe the enterotype patterns of stool samples. Two enterotypes were identified, which were dominated by Treponema (enterotype 1) and Prevotella (enterotype 2), respectively (Fig. 2a). The violin plots illustrated the relative abundances of dominant bacteria between the two enterotypes (Fig. 2b). In addition, the relationship of the enterotypes with porcine feeding behavior traits is shown in Fig. 2c. The Prevotella-predominant enterotype (cluster 2) had a significantly higher ADFI value than the Treponema-enterotype $(P=0.01)$. However, the enterotype-like clusters were not significantly associated with the ADET or ADEV $(P>0.05$, Fig. 2c).

\section{Association of fecal bacteria with porcine feeding behavior}

A total of 34 OTUs were significantly associated with the ADFI in the two-part model analysis (FDR $<0.05$, Fig. 3a). Eighteen out of the 34 OTUs had strongly positive correlations with the ADFI, while the other 16 OTUs showed significantly negative associations (Fig. 3a and Additional file 4: Table S2). Of the 18 positive associations, 12 OTUs were annotated to Prevotella, and the other six OTUs were separately assigned to Lachnospiraceae, Faecalibacterium prausnitzii, Ruminococcaceae, S24-7, Anaeroplasma and Sutterella. Among the 16 negative correlations, eight OTUs were annotated to the family level, including two OTUs annotated to Ruminococcaceae, two OTUs to RFP12, and one OTU to each of Christensenellaceae, Dethiosulfovibrionaceae, Elusimicrobiaceae and Veillonellaceae. Five OTUs were assigned to the genus level, including two OTUs to CF231, and the other three OTUs to Sphaerochaeta, YRC22 and Lactobacillus. Only OTU386 was annotated to the species Butyricicoccus pullicaecorum. However, at the threshold of FDR $<0.05$, we didn't detect any OTUs significantly associated with the ADET or ADEV.

We also employed randomForest for regression analysis to further evaluate the association between fecal bacteria and the ADFI. A total of 15 OTUs were identified to significantly associate the ADFI. These OTUs were annotated to the bacterial taxa similar to those identified by two-part model, suggesting the repeatability of the association results (Fig. $3 \mathrm{~b}$ ). Additionally, we also identified two OTUs for each of the ADET and ADEV by randomForest analysis (Additional file 5: Figure S3).

To determine the hub OTUs related to the ADFI, the 34. OTUs detected by the two-part model were used to
A

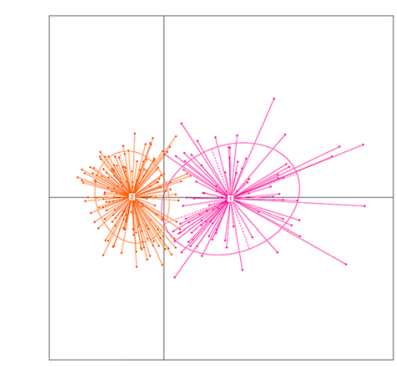

C

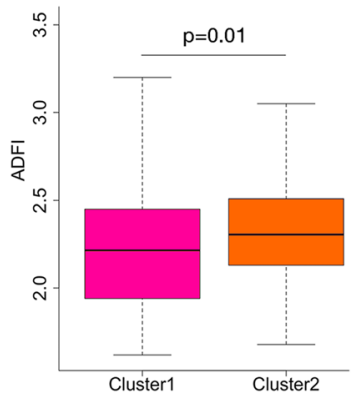

B
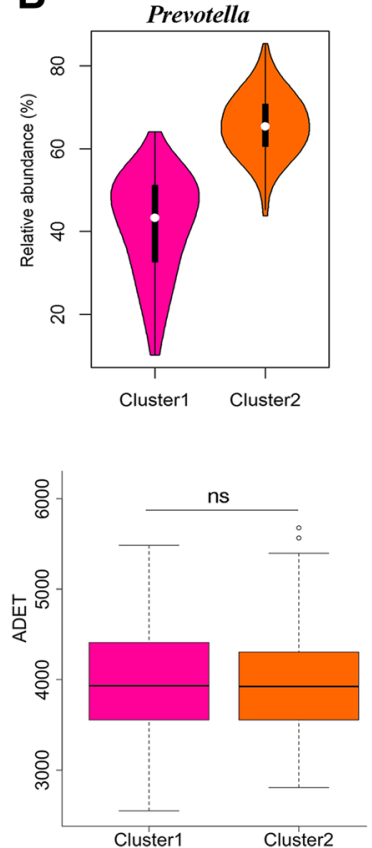
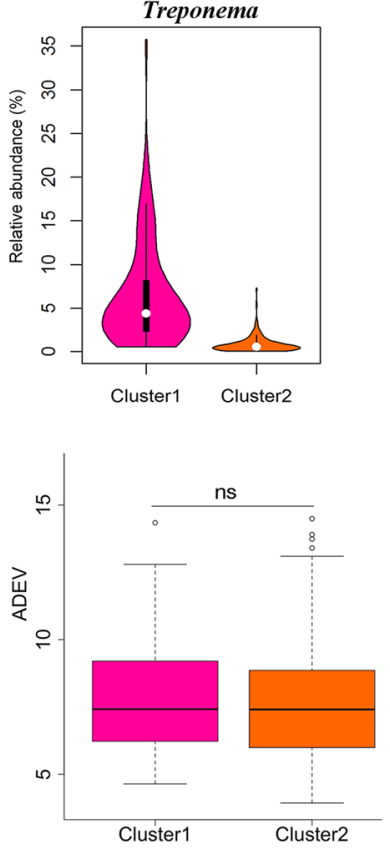

Fig. 2 Enterotype-like cluster distribution in 280 experimental pigs. a Enterotype-like group assignation of experimental pigs. Enterotype 1 was dominated by Treponema, and enterotype 2 was dominated by Prevotella. b Relative abundance of Treponema and Prevotella in each enterotype. c Association of enterotype-like clusters with the ADFI, ADET and ADEV 


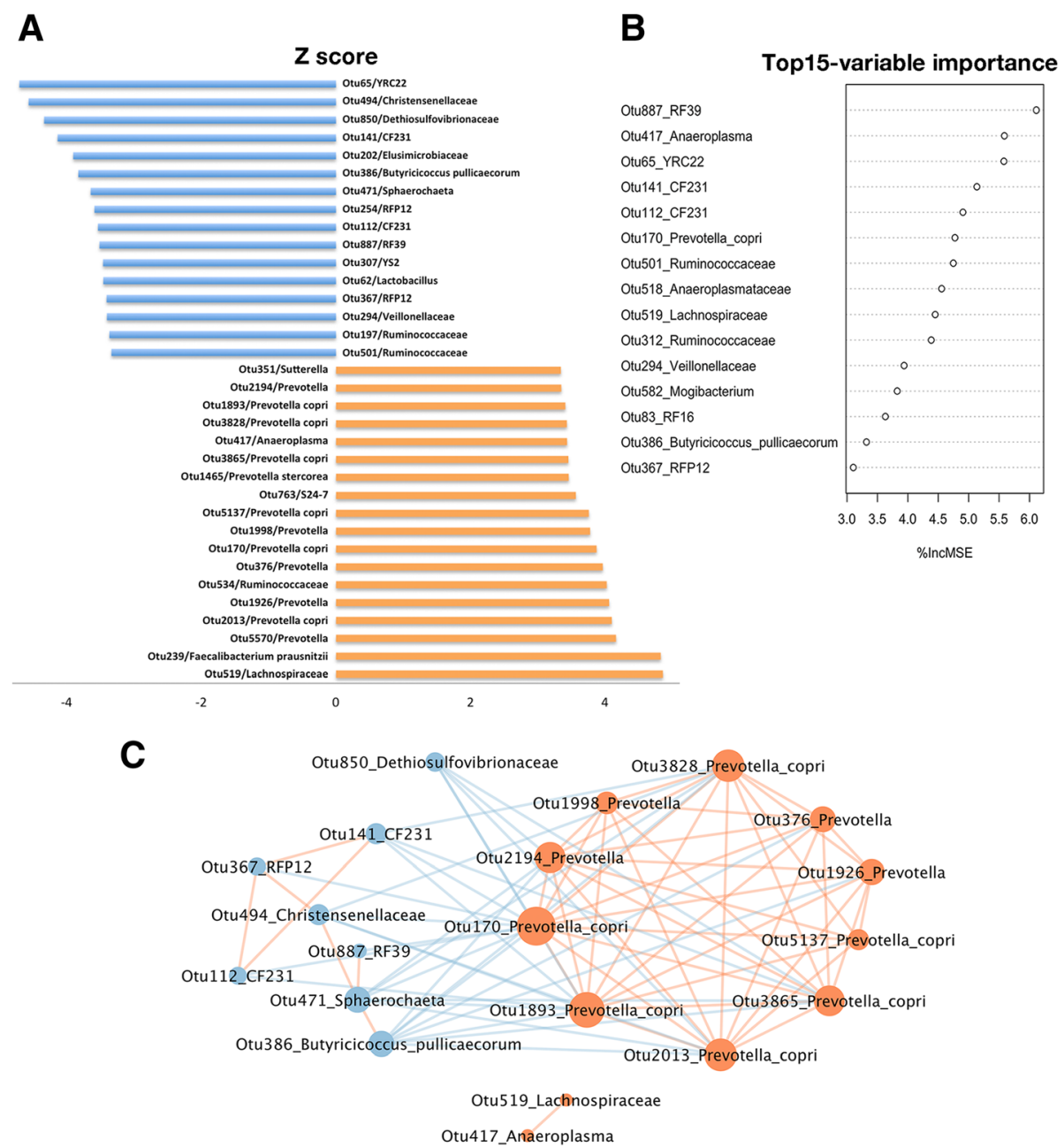

Fig. 3 Porcine average daily feed intake (ADFI)-associated OTUs identified in this study and its interaction network. a The 34 ADFI-associated OTUs identified by two-part model (FDR $<0.05)$ are shown as Z scores. Blue bars show the negative associations and orange bars indicate the positive associations. $\mathbf{b}$ The top informative OTUs related to the ADFI detected by randomForest analysis. c Co-abundance network analysis of the ADFl-associated OTUs reveals the significant interactions. The orange nodes correspond to the OTUs showing the positive association with the $\mathrm{ADFl}$, and the blue nodes represent the OTUs negatively associated with the ADFI. Edge color indicates positive (orange) and negative (blue) correlations. The size of nodes represents the degree of connectivity

estimate sparse correlations using the SparCC method [18]. Only OTU170 (Prevotella copri) had the connectivity with all other nodes (Fig. 3c). We also observed that those OTUs positively associated with the ADFI and annotated to Prevotella were clustered into a sub-module that was negatively correlated with the OTUs showing negative effect on the ADFI. Combining the results of the association study and the co-abundance network analysis, we suggested that Prevotella, especially Prevotella copri should play a core effect on porcine feed intake.

\section{Contribution of the gut bacteria to the pen effect on the ADFI}

To test the hypothesis that the pen effect on the ADFI values might be associated with the stochastic differences in the bacterial exchange and colonization between pigs in the same pen and different pens, we focused on those OTUs that belonged to the 34 ADFI-associated OTUs and showed significant difference of the relative abundances between pigs in different pens. As the result, the relative abundances of five ADFI-associated OTUs (Otu351, Otu494, Otu202, OTU367 and Otu294) were significantly influenced by pens (Additional file 6: Table S3). We next treated the relative abundance of the OTU351, OTU494 and OTU202 as the fixed effect in the linear regression analysis, and re-evaluated the effect of pens on the ADFI values. Interestingly, the significant difference of the ADFI values in pigs in different pens was indeed vanished $(P=0.07)$, suggesting that the large variation of the phylogenetic composition of gut microbiota in pigs housed in different pens caused the pen effect on the ADFI. 


\section{Predicted function capacities of gut microbiome related to porcine ADFI}

We predicted functional capacity profiling using $16 \mathrm{~S}$ rRNA marker gene sequences to identify potential function categories of gut microbiome related to porcine ADFI by PICRUST software [19]. At the significance threshold of FDR $<0.05$, we identified 204 KEGG Orthologies (KOs) showing significant associations with the ADFI (Additional file 7: Table S4). These ADFI-associated KOs were enriched in the pathways related to amino acid biosynthesis and metabolism (cysteine and methionine metabolism, histidine metabolism, phenylalanine, tyrosine and tryptophan biosynthesis, and alanine, aspartate and glutamate metabolism), and sugar metabolism (such as amino sugar and nucleotide sugar metabolism, starch and sucrose metabolism, and fructose and mannose metabolism) (Additional file 8: Figure S4). Interestingly, the KOs negatively associated with the ADFI were enriched in the pathways of the biosynthesis of branched chain amino acids (valine, leucine and isoleucine), histidine, tryptophan, arginine and proline, while the KOs positively associated with the ADFI were enriched in the biosynthesis of serine, cysteine and methionine (Fig. 4a). We further evaluated the contribution of the ADFI-associated bacterial taxa to the changes of the ADFI-associated pathways related to amino acid biosynthesis and metabolism. Those bacterial taxa increasing porcine ADFI, especially Prevotella and Faecalibacterium prausnitzii, were positively associated with the KOs enriched in the biosynthesis of serine, cysteine and methionine, but negatively correlated with those KOs negatively associated with the ADFI $(P<0.001)$. On the contrary, the bacterial taxa decreasing porcine ADFI showed the inverse correlations with the ADFI-associated KOs (Fig. 4b).

\section{Discussion}

The role of gut microbiota in the regulation of host physiological functions in both health and disease state is a hot topic in the research field of gut microbiome. Researchers have summarized the possible role of gut microbiota in host appetite control [1] and the mechanism of the microbiota-gut-brain axis in regulating host appetite and metabolism [7]. Furthermore, the correlation between the diversity of the gastrointestinal microbial community and cattle feed intake was also investigated [20]. However, to our knowledge, there is no study identifying gut microbes associated with porcine appetite until now. In the present study, we uncovered the potential relationships between porcine feeding behavior traits including average daily feed intake, average daily eating visits and average daily eating time, and gut bacterial taxa by $16 \mathrm{~S}$ rRNA gene sequencing analysis.
We also revealed potential function capacities of gut microbiome related to porcine ADFI.

The ADFI was positively associated with backfat thickness, average daily gain and residual feed intake, suggesting that increasing feed intake not only promotes growth, but also increases fat deposition, but possibly decreases feed efficiency. A total of 34 OTUs were identified to associate porcine ADFI, but we did not identify any OTUs associated with the ADET or ADEV in this study. Moreover, the phenotypic values of eating visits and eating time were not correlated with feed intake. The main explanation for this observation should be the reason that it was difficult to accurately record porcine eating time and visiting times, because some pigs did not intake the feed when they visited the automatic feeding trough or the pigs only felt funny for out and in of the machine.

Some SCFA-producing bacteria showed negative correlations with feed intake (Fig. 3a). For examples, Christensenellaceae (OTU494) is Gram-negative bacteria, and can produce the SCFAs [21]. Goodrich et al. [22] revealed that Christensenellaceae might be a marker for low BMI in humans. This was concordance with the finding in this study that the ADFI was positively associated with backfat thickness. Butyricicoccus pullicaecorum (OTU386) is an anaerobic and butyrate-producing bacterium [23]. Veillonellaceae (OTU294) that contains the genus Mitsuokella, Megasphaera and Acidaminococcus, could utilize dietary amino acids [24] and produce high amounts of the SCFAs [25]. Higher abundance of Ruminococcaceae (OTU197 and OTU501) was found in the low-ADFI pigs. Some species of Ruminococcaceae (e.g. Ruminococcus flavefaciens and Ruminococcus albus) can produce lactate and propionate [26]. SCFAs have an important effect on regulating host energy metabolism and appetite. Locally in the gut, SCFAs can directly facilitate the release of the anorexigenic hormones PYY and GLP-1 from L-cells [27]. Chambers et al. [28] showed that there was an increase in the hormones PYY and GLP-1, and a decrease in energy intake when delivering propionate directly to the colon. In addition, Lactobacillus (OTU62) was detected to negatively associate the ADFI in this study. Lactobacillus has always been used as probiotics and can produce lactic acid to promote intestinal development and metabolism [29]. High level of lactate can activate the satiation pathway and reduce food intake [30]. Pessione et al. [31] summarized that biogenic amines from lysine, phenylalanine, histidine and tyrosine are released by decarboxylation of lactic acid bacteria. Correspondingly, in the predicted function capacity analysis of gut microbiome based on 16S rRNA gene sequencing data, the genes involved in the metabolism and biosynthesis of histidine, arginine, proline, branched chain amino acids, and aromatic 


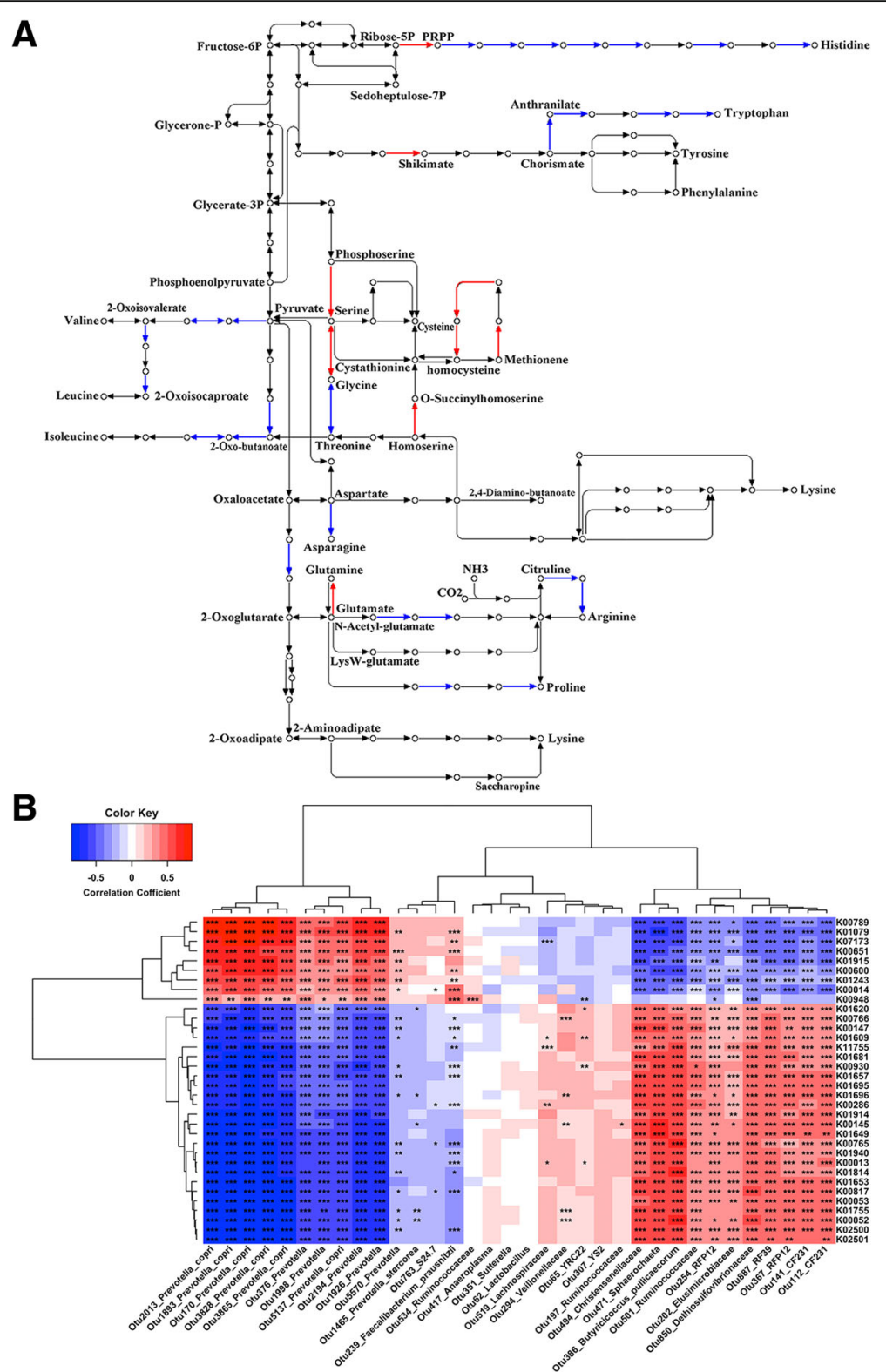

Fig. 4 The amino acid biosynthesis pathway enriched by the ADFl-associated KEGG Orthologies (KO) and its correlation with the ADFI-associated OTUs. a The amino acid biosynthesis pathway enriched by the ADFI-associated KOs. Red lines represent positive correlations with the ADFI, while blue lines indicate negative correlations with the ADFI. $\mathbf{b}$ The correlation between the ADFI-associated OTUs and the ADFI-associated KOs related to amino acid metabolism. ${ }^{*} P<0.05$, ${ }^{*} P<0.01$ and ${ }^{* *} P<0.001$. The definitions of KOs are listed in Additional file 7: Table S4

amino acids (tryptophan, phenylalanine and tyrosine) were increased along with the declining feed intake. The activity of histamine neurons could control energy balance and behavioral responses. Interestingly, feeding a low protein diet added with lysine, methionine and tryptophan to piglets reduced feed intake and body weight gain [32]. Batterham et al. [33] also reported that protein inhibits food intake and appetite more than carbohydrate or fat in obese individuals. The increased concentration of branched amino acid in the plasma significantly increased the release of some gut hormones (e.g. GLP-1 and insulin) [34]. Oral administration of phenylalanine reduced food intake in diet-induced obese mice [35]. Especially, tryptophan significantly increased plasma concentrations of PYY and cholecystokinin that are both regarded as satiation signal [36]. Holzer [37] indicated that the synthesis of neuropeptides might be affected by microbial control of the availability of amino acids. These results suggested that some gut bacteria might suppress porcine feed intake through producing SCFAs and lactic acid, and regulating the metabolism of amino acids. However, compared to the functional prediction based on 16S rRNA sequencing data, a follow-up 
metagenomic sequencing analysis in the future study would help better to elucidate the function and mechanism of gut microbiota suppressing feed intake.

In agreement with the previous report [38], Prevotella was the hub in the interaction network of the ADFI-associated OTUs. In the enterotype analysis, we identified that the Prevotella-enterotype had a greater ADFI than the Treponema enterotype-like cluster. Myer et al. [20] also showed that Prevotella had a higher abundance in the high ADFI group than in the low ADFI individuals in beef cattle. A previous report indicated that Prevotella is in connection with an increased long-term carbohydrate intake [39]. Prevotella that is capable of metabolizing complex dietary polysaccharides may favor the uptake of monosaccharides in the host gut [38]. The ADFI-associated OTUs annotated to Prevotella were significantly associated with the ADFI-associated KOs. Queipo-Ortuno et al. [40] reported that the abundance of Prevotella was positively correlated with serum ghrelin which is the only appetizing hormone known at present. Taken together, we speculated that Prevotella (particularly Prevotella copri) might be a critical bacterial taxon stimulating the feed intake.

\section{Conclusion}

The present study showed that some bacteria producing SCFAs and lactic acid (e.g. Ruminococcaceae and Lactobacillus) might play an important role in suppressing porcine feed intake, while Prevotella could promote porcine feed intake and might be the keystone bacteria for host appetite control. These results suggested that the gut microbial community might have an important contribution to porcine feeding behavior. The modulation of gut microbiota could be benefit for the control of feed intake in pig industry.

\section{Methods}

Experimental animals and phenotypic measurement

A total of 280 Duroc pigs (111 females and 169 males) were used in this study. All experimental pigs were healthy and not received antibiotics, probiotics or prebiotics during the period of experiment. Feeding and management of all experimental pigs were described in detail in our previous study [14]. Briefly, all experimental pigs were weaned at the age of 28 days, and then raised in the nursery house until its body weight achieved $30 \mathrm{~kg}$. After that, experimental pigs were transferred to the fattening house where automatic feeding troughs (Osborne Industries, USA) were installed. Male and female pigs were separately housed in different pens. The same formula feed and clean water were available ad libitum for all experimental pigs. Phenotypic performances of experimental pigs at the stage of fattening from $30 \mathrm{~kg}$ (at the age of $70 \sim 90$ days) to $100 \mathrm{~kg}$ body weight (at the age of $170 \sim 190$ days) were measured by the instrument of automatic feeding trough (Osborne Industries, USA). When their body weight achieved $100 \mathrm{~kg}$, the experimental pigs were slaughtered at a commercial slaughterhouse by bleeding after electrical stunning. To avoid the effect of environmental adaptation of experimental pigs with automatic feeding trough, we used the phenotypic values obtained from day 100 to 160 (intermediate stage of measurement) for further analysis. ADFI, ADET and ADEV were used as the traits evaluating feeding behavior and appetite. As the phylogenetic composition and microbial ecosystem of gut microbiota remain relatively stable after maturation [41], we collected the fecal samples from all 280 experimental pigs at the age of 140 days (within the interval of 100-160 days). All fecal samples were harvested from animal's anus, and then immediately dipped in liquid nitrogen. After transported to the laboratory, the samples were transferred into $-80^{\circ} \mathrm{C}$ freezer until use.

\section{Evaluating the environmental and host effects on phenotypic values of feeding behavior}

The effects of pen and host kinship on phenotypic values of feeding behavior were evaluated by comparing the similarity of phenotypic values between the pigs in the same pen or in the full-sib members, and the pigs in different pens or in unrelated individual groups. $T$-test was used to compare the phenotypic values between male and female pigs.

\section{$16 \mathrm{~S}$ rRNA gene sequencing and data processing}

Microbial DNA was extracted from feces with QIAamp Fast DNA Stool Mini Kit (Qiagen, Germany) according to the manufacturer's protocol. The V4 region of the $16 \mathrm{~S}$ rRNA gene was amplified using the barcode fusion primers 515F (5'-GTGCCAGCMGCCGCGGTAA-3') and 806R (5'-GGACTACHVGGGTWTCTAAT-3'). The PCR products were used to construct the libraries, and then sequenced using the paired-end method on Illumina MiSeq platform (Illumina, USA). Data processing was performed by the standard protocols of bioinformatics analysis for $16 \mathrm{~S}$ rRNA gene sequencing. Firstly, the primers, barcode sequences, and low quality reads were removed from raw data [42]. High-quality paired-end clean sequence reads were then assembled into tags using FLASH (v.1.2.11). And then, USEARCH software (v7.0.1090) was used to pick OTUs at 97\% similarity [43]. Taxonomic assignments for the aligned sequences were made using the Ribosomal Database project (RDP) classifer program (v2.2) [44]. Those OTUs that were presented in less than $5 \%$ of the experimental pigs and had relative abundance $<0.01 \%$ were removed from further analysis. 


\section{Enterotype analysis}

According to the method described by Arumugam et al. [17], we analyzed the enterotype of the experimental pig cohort. In brief, the clustering of samples was performed by using the Partitioning Around Medoids (PAM) clustering algorithm and Jensen-Shannon divergence (JSD) distance based on the relative abundances of bacteria at the genus level. And then, the Calinski-harabasz $(\mathrm{CH})$ Index was used to evaluate the optional number of clusters from 2 to 20 clusters. When the cluster number had the highest $\mathrm{CH}$ index and was also validated by the silhouette coefficient, it was set as the optimal number. The possible effect of predicted enterotype-like clusters on the host feeding behavior was tested by $t$-test.

\section{Association study}

Because of the non-normal distribution of the relative abundances of most bacteria in the experimental pigs, a two-part model was applied to analyze the association of bacteria with the traits of feeding behavior as used in previous study [45]. Briefly, the two-part model accounts for both binary and quantitative features. The binary analysis was to test for the effect of the presence/absence of the microbes on porcine feeding behavior, and the quantitative model analyzed for association between the abundance of the detected microbes and the host feeding behavior. To further evaluate the effect of both binary and quantitative features, a meta-analysis was performed using an unweighted $\mathrm{Z}$ method. The minimum $P$-value of binary, quantitative and meta-analysis was set as the final association $P$-value. The $Z$-score was calculated based on the $\mathrm{Z}$ distribution. In addition, $1000 \times$ permutation test was used to control the false discovery rate (FDR). The FDR $\leq 0.05$ was set as the significant threshold. The residuals of ADFI, ADET and ADEV values corrected the effect of sex were used for association analysis. We also used the package of randomForest for regression to further confirm the association results [46]. The variable importance was estimated using the index of increase in Mean Squared Error (\%incMSE). The \%incMSE value above 3 was considered as an important variable.

\section{Network analysis for the ADFI-associated OTUs}

To identify the hub OTUs from the ADFI-associated OTUs detected by two-part model, OTU networks were predicted using the SparCC approach as described by Friedman et al. [18]. In brief, we first calculated the SparCC correlation coefficient matrix for the ADFI-associated OTUs with SpiecEasi package [47]. And then, the network analysis was performed and visualized using cytoscape at the absolute correlation coefficient threshold of 0.3 [48].

\section{Prediction of functional capacity of gut microbiome}

To predict functional capacity of the gut microbiome, PICRUST (v1.0.0) was applied to calculate the relative abundances of KEGG pathways according to the $16 \mathrm{~S}$ rRNA gene sequences [19]. Correlations between ADFI values and relative abundances of KOs were implemented with MaAsLin. The association between the ADFI-associated OTUs detected by two-part model and the ADFI-related KOs was evaluated by spearman correlation analysis with $\mathrm{R}$ software.

\section{Additional files}

Additional file 1: Figure S1. Distribution of phenotypic values of porcine feeding behavior-related traits. The phenotypic values of feeding behavior-related traits were basically in a normal distribution. The name of each trait was shown on the top: Average daily feed intake (ADFI), average daily eating time (ADET) and average daily eating visits (ADEV) (TIF 1374 kb)

Additional file 2: Figure S2. Factors affecting porcine feeding behavior traits. (A) Sex had no significant effect on porcine feeding behavior traits. (B) Pen showed significant effect on porcine feeding behavior traits. (C) Comparison of porcine feeding behavior traits between full-siblings and unrelated individuals (ns: $P$ value was not achieved significance; ${ }^{* * *} P<0.001$ for student's t-test). (TIF $1241 \mathrm{~kb}$ )

Additional file 3: Table S1. The significant differences of ADFI values between pigs in different pens detected by pairwise comparison. (XLS 30 kb)

Additional file 4: Table S2. The OTUs significantly associated with the ADFI by the two-part model (FDR <0.05). (XLS $44 \mathrm{~kb}$ )

Additional file 5: Figure S3. The top informative OTUs for ADET (A) and ADEV (B) detected by randomForest analysis. (TIF $529 \mathrm{~kb}$ )

Additional file 6: Table S3. Five OTUs out of the 34 ADFI-associated OTUs whose relative abundances were significantly influenced by pens. (XLS $33 \mathrm{~kb}$ )

Additional file 7: Table S4. The correlation between the relative abundance of KOs and the ADFI by MaAsLin. (XLS $65 \mathrm{~kb}$ )

Additional file 8: Figure S4. The top 25 pathways enriched by the ADFI-associated KEGG Orthologies (KOs). The data on the bars indicates the KO numbers. (TIF $2160 \mathrm{~kb}$ )

Additional file 9: Table S5. The ARRIVE Guidelines Checklist. (DOC 59 kb)

\section{Abbreviations}

ADET: Average daily eating time; ADEV: Average daily eating visits; ADFI: Average daily feed intake; ADG: Average daily gain; AgRP: Agoutirelated peptide; ARC: Hypothalamic arcuate nucleus; BF: Backfat; $\mathrm{CH}$ : Calinski-harabasz index; FDR: False discovery rate; GLP-1: Glucagonlike peptide 1; JSD: Jensen-Shannon divergence distance; KEGG: Kyoto Encyclopedia of Genes and Genomes; MSE: Mean squared error; NPY: Neuropeptide tyrosine; OTU: Operational taxonomic units; PAM: Partitioning around medoids; POMC: Proopiomelanocortin; PYY: Peptide YY; RFI: Residual feed intake; rRNA: Ribosomal Robonucleic Acid; SCFA: Short-chain fatty acid

\section{Acknowledgements}

We thank the colleagues in the State Key Laboratory of Pig Genetic Improvement and Production Technology, Jiangxi Agricultural University for their efforts in the management of experimental pig populations.

\section{Funding}

This study was funded by National Natural Science Foundation of China (grant number 31472071 and 31702103). Hui Yang was supported by National Postdoctoral Program for Innovative Talents (No. BX201700102). The funding bodies had no role in the design of the study and collection, analysis, and interpretation of data and in writing this manuscript. 


\section{Availability of data and materials}

All 165 rRNA gene sequencing data was submitted to the NCBI with the accession number PRJNA356465.

\section{Authors' contributions}

$\mathrm{LH}$ and $\mathrm{CC}$ designed the project and revised the manuscript. HY performed the experiment, analyzed the data and wrote the manuscript. MY measured phenotypes. SF, XH, MH analyzed the data. SK and HF collected the fecal samples. JG, JW and YZ extracted DNA from fecal samples. All authors read and approved the final manuscript.

\section{Ethics approval}

All animal works were conducted according to the guidelines for the care and use of experimental animals established by the Ministry of Agriculture of China. The project was approved by Animal Care and Use Committee (ACUC) in Jiangxi Agricultural University. The ARRIVE Guidelines Checklist was in the Additional file 9: Table S5.

\section{Consent for publication}

Not applicable.

\section{Competing interests}

The authors declare that they have no competing interests.

\section{Publisher's Note}

Springer Nature remains neutral with regard to jurisdictional claims in published maps and institutional affiliations.

\section{Author details}

'State Key Laboratory of Pig Genetic Improvement and Production Technology, Jiangxi Agricultural University, Nanchang 330045, China. ${ }^{2}$ College of Bioscience and Engineering, Jiangxi Agricultural University, Nanchang, China. ${ }^{3}$ National Engineering Research Center for Breeding Swine Industry, Guangdong Wens Foodstuff Co. Ltd., Xinxing, China.

Received: 8 June 2018 Accepted: 2 December 2018

\section{Published online: 14 December 2018}

\section{References}

1. Fetissov SO. Role of the gut microbiota in host appetite control: bacterial growth to animal feeding behaviour. Nat Rev Endocrinol. 2017;13(1):11-25.

2. Keen-Rhinehart E, Ondek K, Schneider JE. Neuroendocrine regulation of appetitive ingestive behavior. Front Neurosci. 2013;7:213.

3. Sasaki T, Matsui S, Kitamura T. Control of appetite and food preference by NMDA receptor and its co-agonist d-serine. Int J Mol Sci. 2016;17(7). https:// doi.org/10.3390/ijms17071081.

4. Schwartz MW, Woods SC, Porte D Jr, Seeley RJ, Baskin DG. Central nervous system control of food intake. Nature. 2000;404(6778):661-71.

5. Simon JJ, Skunde M, Walther S, Bendszus M, Herzog W, Friederich HC Neural signature of food reward processing in bulimic-type eating disorders. Soc Cogn Affect Neurosci. 2016;11(9):1393-401.

6. Munzberg H, Qualls-Creekmore E, Yu S, Morrison CD, Berthoud HR. Hedonics act in unison with the homeostatic system to unconsciously control body weight. Front Nutr. 2016;3:6.

7. van de Wouw M, Schellekens H, Dinan TG, Cryan JF. Microbiota-gut-brain Axis: modulator of host metabolism and appetite. J Nutr. 2017;147(5):727-45.

8. Smith MI, Yatsunenko T, Manary MJ, Trehan I, Mkakosya R, Cheng J, et al. Gut microbiomes of Malawian twin pairs discordant for kwashiorkor. Science. 2013;339(6119):548-54.

9. Turnbaugh PJ, Gordon J. The core gut microbiome, energy balance and obesity. J Physiol. 2009;587(Pt 17):4153-8.

10. Morita C, Tsuji H, Hata T, Gondo M, Takakura S, Kawai K, et al. Gut Dysbiosis in patients with anorexia nervosa. PLoS One. 2015:10(12):e0145274.

11. Zhang C, Li S, Yang L, Huang P, Li W, Wang S, et al. Structural modulation of gut microbiota in life-long calorie-restricted mice. Nat Commun. 2013:4:2163.

12. Breton J, Tennoune N, Lucas N, Francois M, Legrand R, Jacquemot J, et al. Gut Commensal E. coli proteins activate host satiety pathways following nutrient-induced bacterial growth. Cell Metab. 2016;23(2):324-34.

13. Janssen S, Depoortere I. Nutrient sensing in the gut: new roads to therapeutics? Trends Endocrinol Metab. 2013;24(2):92-100.
14. Yang $H$, Huang $X$, Fang $S$, He M, Zhao $Y$, Wu Z, et al. Unraveling the fecal microbiota and metagenomic functional capacity associated with feed efficiency in pigs. Front Microbiol. 2017:8:1555.

15. Lamendella R, Domingo JW, Ghosh S, Martinson J, Oerther DB. Comparative fecal metagenomics unveils unique functional capacity of the swine gut. BMC Microbiol. 2011;11:103.

16. Looft T, Allen HK, Cantarel BL, Levine UY, Bayles DO, Alt DP, et al. Bacteria, phages and pigs: the effects of in-feed antibiotics on the microbiome at different gut locations. ISME J. 2014;8(8):1566-76.

17. Arumugam M, Raes J, Pelletier E, Le Paslier D, Yamada T, Mende DR, et al. Enterotypes of the human gut microbiome. Nature. 2011;473(7346):174-80.

18. Friedman J, Alm EJ. Inferring correlation networks from genomic survey data. PLoS Comp Biol. 2012;8(9):269-76.

19. Langille MG, Zaneveld J, Caporaso JG, McDonald D, Knights D, Reyes JA, et al. Predictive functional profiling of microbial communities using $16 \mathrm{~S}$ rRNA marker gene sequences. Nat Biotechnol. 2013;31(9):814-21.

20. Myer PR, Freetly HC, Wells JE, Tpl S, Kuehn LA. Analysis of the gut bacterial communities in beef cattle and their association with feed intake, growth, and efficiency. J Anim Sci. 2017;95(7):3215.

21. Morotomi M, Nagai F, Watanabe Y. Description of Christensenella minuta gen. nov., sp. nov., isolated from human faeces, which forms a distinct branch in the order Clostridiales, and proposal of Christensenellaceae fam. nov. Int J Syst Evol Microbiol. 2012;62(Pt 1):144-9.

22. Goodrich JK, Waters JL, Poole AC, Sutter JL, Koren O, Blekhman R, et al. Human genetics shape the gut microbiome. Cell. 2014;159(4):789-99.

23. Eeckhaut V, Van IF, Teirlynck E, Pasmans F, Fievez V, Snauwaert C, et al. Butyricicoccus pullicaecorum gen. nov., sp. nov., an anaerobic, butyrateproducing bacterium isolated from the caecal content of a broiler chicken. Int J Syst Evol Microbiol. 2008:58(Pt 12):2799.

24. Dai ZL, Zhang J, Wu G, Zhu WY. Utilization of amino acids by bacteria from the pig small intestine. Amino Acids. 2010;39(5):1201-15.

25. Gamage H, Tetu SG, Chong RWW, Ashton J, Packer NH, Paulsen IT. Cereal products derived from wheat, sorghum, rice and oats alter the infant gut microbiota in vitro. Sci Rep. 2017:7(1):14312

26. Boonsaen P, Kinjo M, Sawanon S, Suzuki Y, Koike S, Kobayashi Y. Partial characterization of phylogeny, ecology and function of the fibrolytic bacterium Ruminococcus flavefaciens OS14, newly isolated from the rumen of swamp buffalo. Anim Sci. 2017. https://doi.org/10.1111/asj.12927.

27. Dumoulin V, Moro F, Barcelo A, Dakka T, Cuber JC. Peptide YY, glucagon-like peptide-1, and neurotensin responses to luminal factors in the isolated vascularly perfused rat ileum. Endocrinology. 1998;139(9):3780-6.

28. Chambers ES, Alexander V, Arianna P, Morrison DJ, Murphy KG, ZacVarghese SEK, et al. Effects of targeted delivery of propionate to the human colon on appetite regulation, body weight maintenance and adiposity in overweight adults. Gut. 2015;64(11):1744-54

29. Song JJ, Tian WJ, Kwok LY, Wang YL, Shang YN, Menghe B, et al. Effects of microencapsulated lactobacillus plantarum LIP-1 on the gut microbiota of hyperlipidaemic rats. Br J Nutr. 2017;118(7):481.

30. Silberbauer CJ, Surina-Baumgartner DM, Arnold M, Langhans W. Prandial lactate infusion inhibits spontaneous feeding in rats. Am J Physiol Regul Integr Comp Physiol. 2000;278(3):R646-53.

31. Pessione $E$, Cirrincione $S$. Bioactive molecules released in food by lactic acid Bacteria: encrypted peptides and biogenic amines. Front Microbiol. 2016;7:876.

32. Luo Z, Li C, Cheng Y, Hang S, Zhu W. Effects of low dietary protein on the metabolites and microbial communities in the caecal digesta of piglets. Arch Anim Nutr. 2015;69(3):212-26.

33. Batterham RL, Cohen MA, Ellis SM, Le Roux CW, Withers DJ, Frost GS, et al. Inhibition of food intake in obese subjects by peptide YY3-36. N Engl J Med. 2003;349(10):941-8.

34. Luscombemarsh ND, Hutchison AT, Soenen S, Steinert RE, Clifton PM, Horowitz M, et al. Plasma free amino acid responses to Intraduodenal whey protein, and relationships with insulin, glucagon-like Peptide-1 and energy intake in lean healthy men. Nutrients. 2016;8(1):4.

35. Alamshah A, Spreckley E, Norton M, Kinseyjones JS, Amin A, Ramgulam A, et al. L-phenylalanine modulates gut hormone release and glucose tolerance, and suppresses food intake through the calcium-sensing receptor in rodents. Int J Obes. 2017;41(11):1693-701.

36. Steinert RE, Ullrich SS, Geary N, Asarian L, Bueter M, Horowitz M, et al. Comparative effects of intraduodenal amino acid infusions on food intake and gut hormone release in healthy males. Physiol Rep. 2017:5(21). https://doi.org/10.14814/phy2.13492. 
37. Holzer P. Neuropeptides, microbiota, and behavior. Int Rev Neurobiol. 2016;131:67.

38. Ramayo-Caldas Y, Mach N, Lepage P, Levenez F, Denis C, Lemonnier G, et al. Phylogenetic network analysis applied to pig gut microbiota identifies an ecosystem structure linked with growth traits. ISME J. 2016;10(12):2973-7.

39. Wu GD, Chen J, Hoffmann C, Bittinger K, Chen YY, Keilbaugh SA, et al. Linking long-term dietary patterns with gut microbial enterotypes. Science. 2011;334(6052):105-8.

40. Queipo-Ortuno MI, Seoane LM, Murri M, Pardo M, Gomez-Zumaquero JM, Cardona F, et al. Gut microbiota composition in male rat models under different nutritional status and physical activity and its association with serum leptin and ghrelin levels. PLoS One. 2013;8(5):e65465.

41. Kim HB, Borewicz K, White BA, Singer RS, Sreevatsan S, Tu ZJ, et al. Longitudinal investigation of the age-related bacterial diversity in the feces of commercial pigs. Vet Microbiol. 2011;153(1-2):124-33.

42. Fadrosh DW, Ma B, Gajer P, Sengamalay N, Ott S, Brotman RM, et al. An improved dual-indexing approach for multiplexed 165 rRNA gene sequencing on the Illumina MiSeq platform. Microbiome. 2014;2(1):6.

43. Majaneva M, Hyytiainen K, Varvio SL, Nagai S, Blomster J. Bioinformatic amplicon read processing strategies strongly affect eukaryotic diversity and the taxonomic composition of communities. PLoS One. 2015;10(6):e0130035.

44. Wang Q, Garrity GM, Tiedje JM, Cole JR. Naive Bayesian classifier for rapid assignment of rRNA sequences into the new bacterial taxonomy. Appl Environ Microbiol. 2007;73(16):5261-7.

45. Fu J, Bonder MJ, Cenit MC, Tigchelaar EF, Maatman A, Dekens JA, et al. The gut microbiome contributes to a substantial proportion of the variation in blood lipids. Circ Res. 2015;117(9):817-24.

46. Kuhn $M$, Leeuw JD, Zeileis A. Building predictive models in $R$ using the caret package. J Stat Softw. 2008;28(5):1-26.

47. Kurtz ZD, Muller CL, Miraldi ER, Littman DR, Blaser MJ, Bonneau RA. Sparse and compositionally robust inference of microbial ecological networks. PLoS Comput Biol. 2015;11(5):e1004226.

48. Shannon P, Markiel A, Ozier O, Baliga NS, Wang JT, Ramage D, et al. Cytoscape: a software environment for integrated models of biomolecular interaction networks. Genome Res. 2003;13(11):2498-504.

Ready to submit your research? Choose BMC and benefit from:

- fast, convenient online submission

- thorough peer review by experienced researchers in your field

- rapid publication on acceptance

- support for research data, including large and complex data types

- gold Open Access which fosters wider collaboration and increased citations

- maximum visibility for your research: over $100 \mathrm{M}$ website views per year

At BMC, research is always in progress.

Learn more biomedcentral.com/submissions 